\title{
Supplementary information of
}

\section{Therapeutic targeting of repurposed anticancer drugs in Alzheimer's Disease: using multi-omics}

approach

\section{Dia Advani ${ }^{1}$, Pravir Kumar', ${ }^{1}$}

Molecular Neuroscience and Functional Genomics Laboratory, Delhi Technological University, Shahabad Daulatpur, Bawana Road, Delhi 110042

$\dagger$ Author to whom correspondence should be addressed:

Pravir Kumar, M.Sc. (BHU), PhD (Germany); PDF/Faculty (USA)

Professor and Head, Department of Biotechnology

Former Dean, Delhi Technological University (Formerly Delhi College of Engineering);

Former Faculty, Neurology Department, Tufts University School of Medicine, Boston MA, USA; Editor, Scientific Reports (Nature Publication); Phone: +91-

9818898622

Email: pravirkumar@dtu.ac.in; kpravir@gmail.com

URL: My NCBI; Google Scholar; ORCID; KUMAR@DTU

Scopus ID: 14831447800; ISI Research ID: B-2164-2015 
Table S1: Functional Enrichment analysis of AD associated genes

\begin{tabular}{|c|c|c|c|c|c|c|c|c|}
\hline DAVID GENOMICS & $\begin{array}{l}\text { DAVID } \\
\text { TRANSCRIPTOMICS }\end{array}$ & $\begin{array}{l}\text { DAVID } \\
\text { METABOLOMIC } \\
\text { S } \\
\end{array}$ & GS2D GENOMICS & $\begin{array}{l}\text { GS2D } \\
\text { TRANSCRIPTOMICS }\end{array}$ & $\begin{array}{l}\text { GS2D } \\
\text { METABOLOMIC } \\
\text { S } \\
\end{array}$ & DAVID combined & GS2D Combined & Common genes \\
\hline APOC1 & AGTR1 & ABCB1 & ABCA7 & CDK5 & ACE & ABCB1 & ABCA7 & ACE \\
\hline APOC2 & ANGPT4 & $\mathrm{ABCG} 2$ & ADAM10 & GFAP & ACHE & $\mathrm{ABCG} 2$ & ACE & ACHE \\
\hline APOE & ATXN1 & ABL1 & APOC1 & NRG1 & ALOX5 & ABL1 & $\mathrm{ACHE}$ & ALOX5 \\
\hline BIN1 & CDK5 & ABL2 & APOE & VSNL1 & APCS & ABL2 & ADAM10 & APOC1 \\
\hline CLU & CSF1 & ACACB & BIN1 & & APLP1 & ACACB & ALOX5 & APOE \\
\hline CR1 & DLG5 & ACADSB & CLU & & APP & ACADSB & APCS & APP \\
\hline EPC2 & DRD4 & $\mathrm{ACE}$ & CR1 & & ATP7B & ACE & APLP1 & BCHE \\
\hline EXOC3L2 & GLI2 & ACHE & EXOC3L2 & & BCHE & ACHE & APOC1 & BIN1 \\
\hline HLA-DRB1 & LTB4R & ACSL5 & MS4A6A & & CAPN1 & ACSL5 & APOE & CDK5 \\
\hline MARK4 & MTHFD1 & ACSM6 & NECTIN2 & & $\mathrm{CH} 25 \mathrm{H}$ & ACSM6 & APP & $\mathrm{CH} 25 \mathrm{H}$ \\
\hline NECTIN2 & NRG1 & ADH1A & PICALM & & CHAT & ADH1A & ATP7B & CHAT \\
\hline PICALM & PPP3CA & ADK & TOMM40 & & COMT & ADK & BCHE & CLU \\
\hline \multirow[t]{26}{*}{ TOMM40 } & PRPF18 & ADRA1A & & & $\mathrm{CP}$ & ADRA1A & BIN1 & COMT \\
\hline & SNCG & AGTR1 & & & CYP2D6 & AGTR1 & CAPN1 & $\mathrm{CP}$ \\
\hline & SORCS3 & AHSG & & & CYP46A1 & AHSG & CDK5 & CR1 \\
\hline & SPAG6 & AKR1E2 & & & DAPK1 & AKR1E2 & $\mathrm{CH} 25 \mathrm{H}$ & CYP2D6 \\
\hline & SPARC & $\mathrm{ALB}$ & & & DRD1 & ALB & CHAT & CYP46A1 \\
\hline & YMEL1 & ALDH18A1 & & & DRD3 & ALDH18A1 & CLU & DAPK1 \\
\hline & & ALDH2 & & & EIF2AK2 & ALDH2 & COMT & DRD1 \\
\hline & & ALOX12 & & & FYN & ALOX12 & $\mathrm{CP}$ & DRD3 \\
\hline & & ALOX15 & & & GAD2 & ALOX15 & CR1 & DRD4 \\
\hline & & ALOX5 & & & GRIN1 & ALOX5 & CYP2D6 & EXOC3L2 \\
\hline & & ALPL & & & GRIN2B & ALPL & CYP46A1 & FYN \\
\hline & & AMY2A & & & GRM5 & AMY2A & DAPK1 & GAD2 \\
\hline & & ANAK7 & & & GSK3B & ANAK7 & DRD1 & GRIN2B \\
\hline & & ANXA11 & & & GST01 & ANGPT4 & DRD3 & GSK3B \\
\hline & & APEX1 & & & GST02 & ANXA11 & EIF2AK2 & GST1 \\
\hline & & APP & & & HFE & APEX1 & EXOC3L2 & GST2 \\
\hline & & ARSA & & & HSPG2 & APOC1 & FYN & HSPG2 \\
\hline & & AS3MT & & & HTR2A & APOC2 & GAD2 & HTR2AQ \\
\hline & & B4GALT1 & & & HTR2C & APOE & GFAP & HTR2C \\
\hline & & CCR1 & & & IDE & APP & GRIN1 & IDE \\
\hline & & CCR2 & & & KCNIP3 & ARSA & GRIN2B & LRP1 \\
\hline & & CCR5 & & & LRP1 & AS3MT & GRM5 & MME \\
\hline & & CELF2 & & & MAOA & ATXN1 & GSK3B & NECTIN2 \\
\hline & & CISD1 & & & MAOB & B4GALT1 & GST01 & NEDD9 \\
\hline & & CRP & & & MAPK8IP1 & $\mathrm{BCHE}$ & GSTO2 & NRG1 \\
\hline & & DNTT & & & MARK1 & BGLAP & HFE & PCDH11X \\
\hline
\end{tabular}




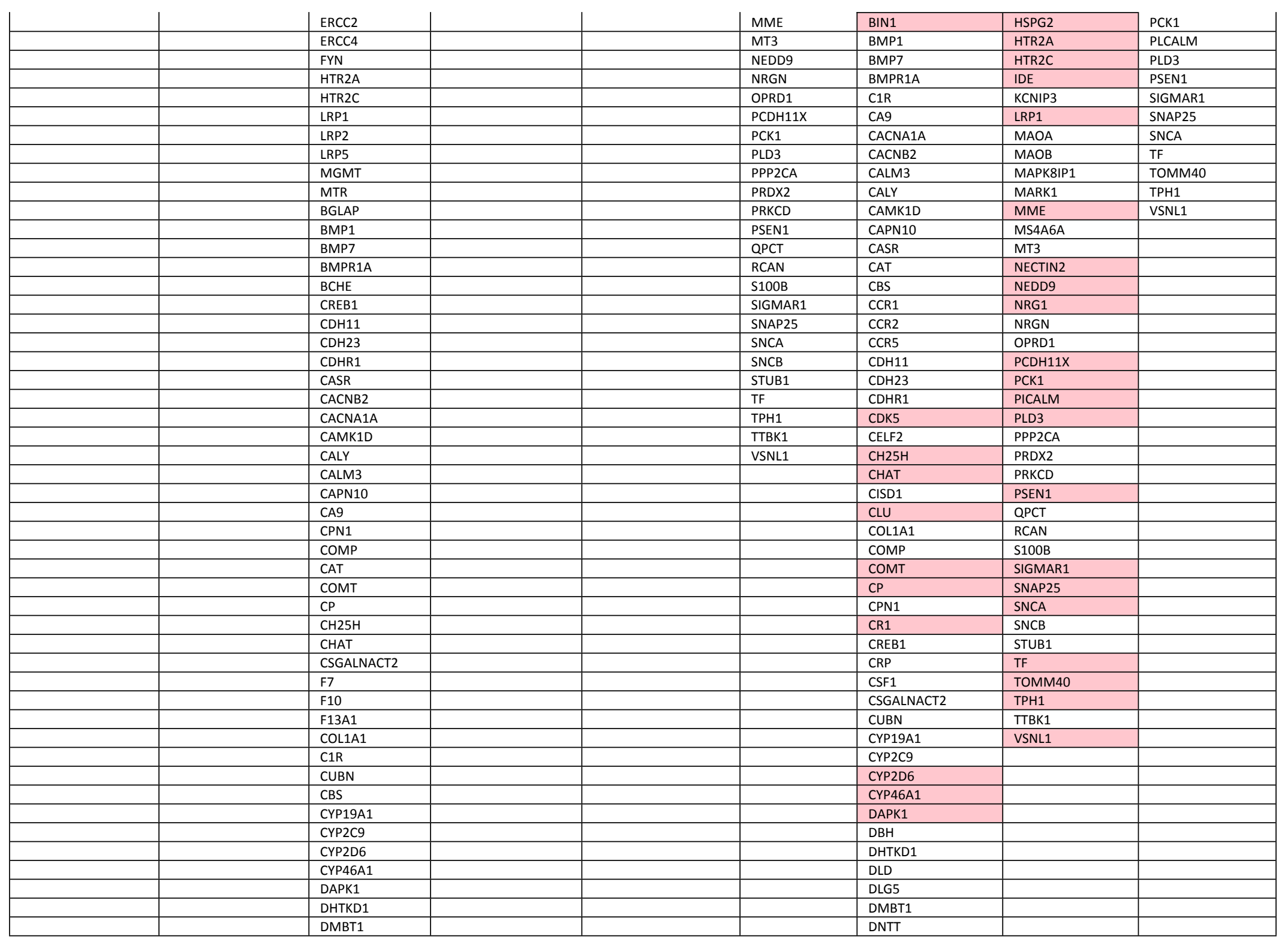




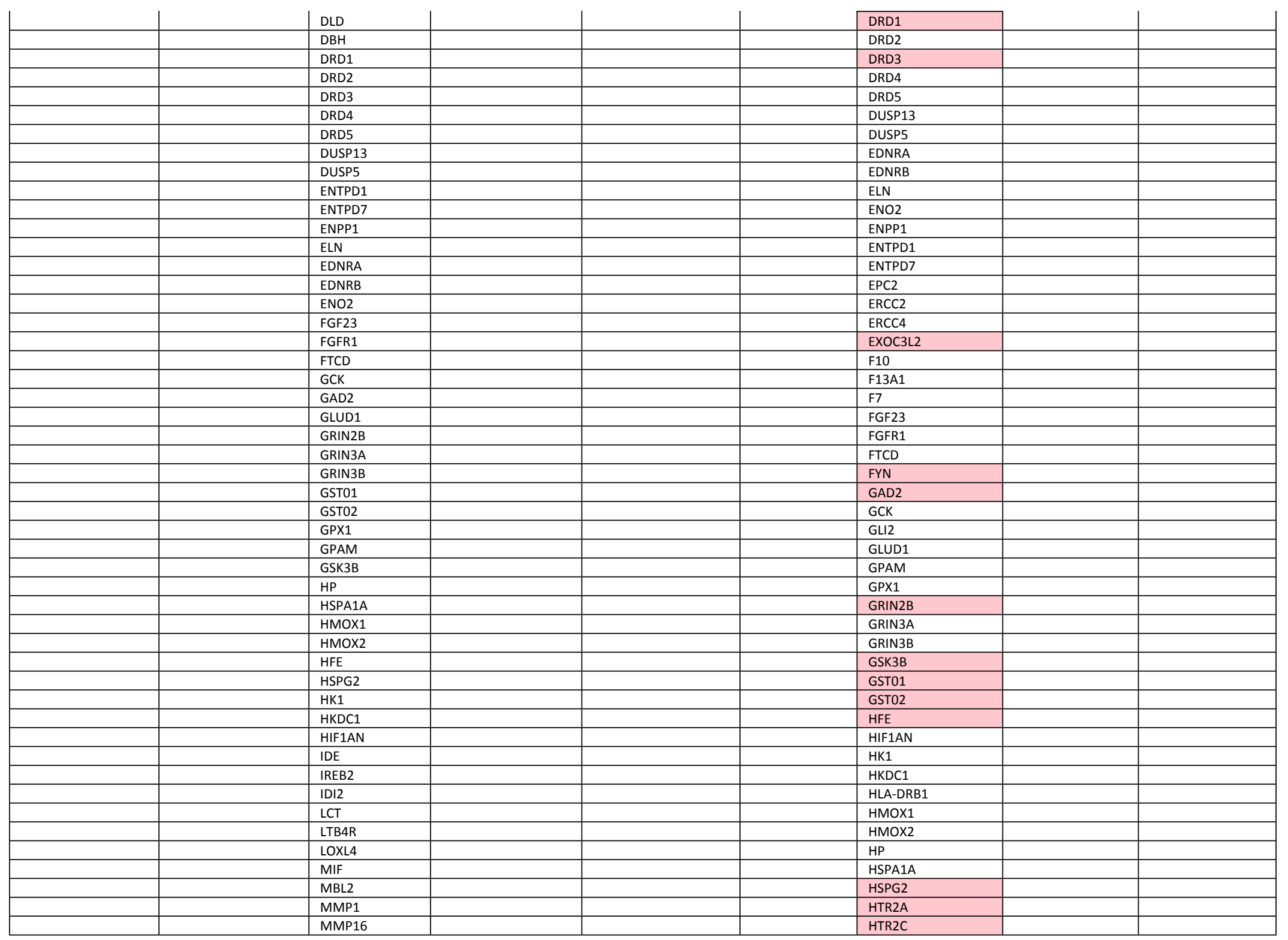

\section{S4}




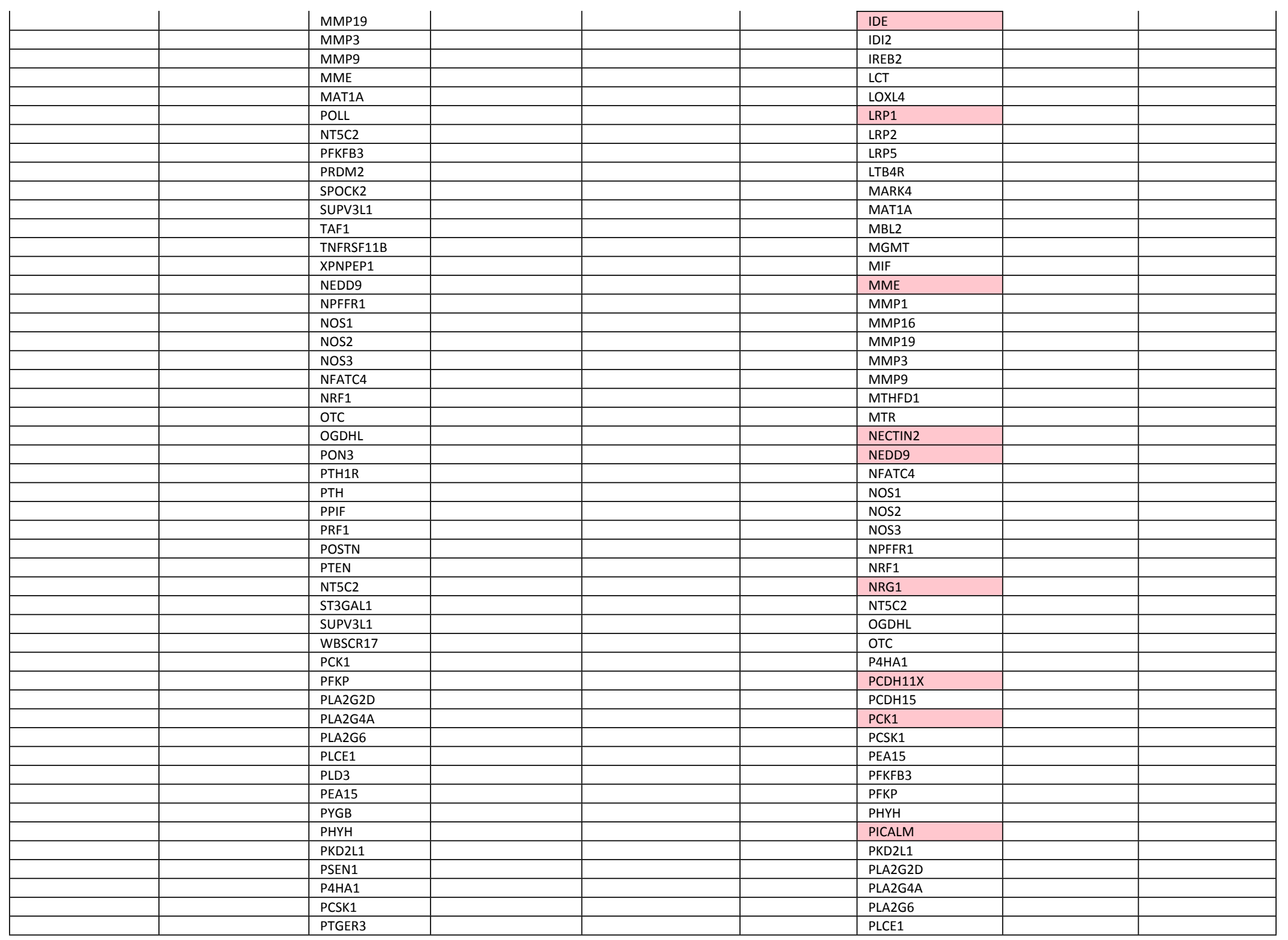




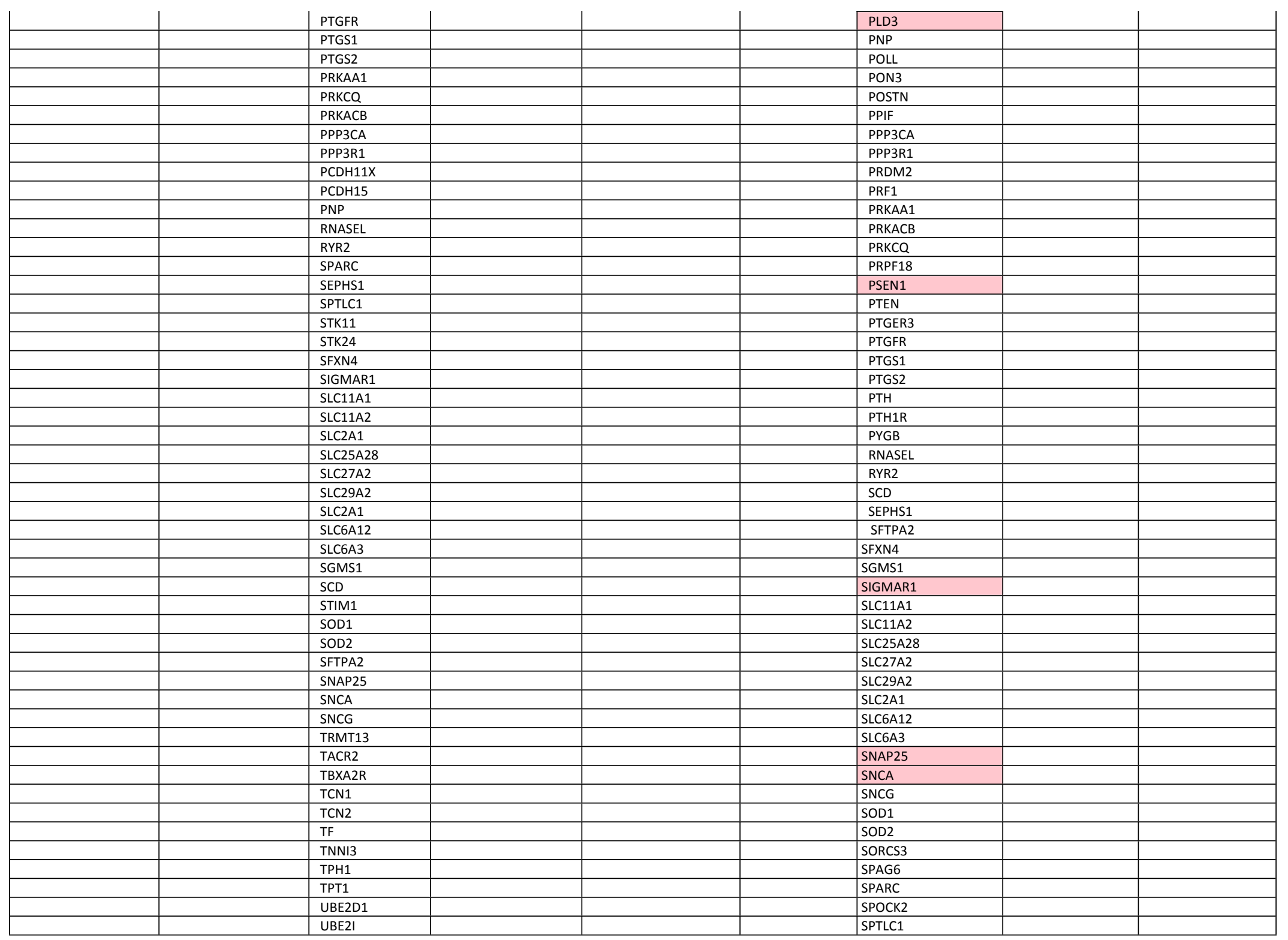




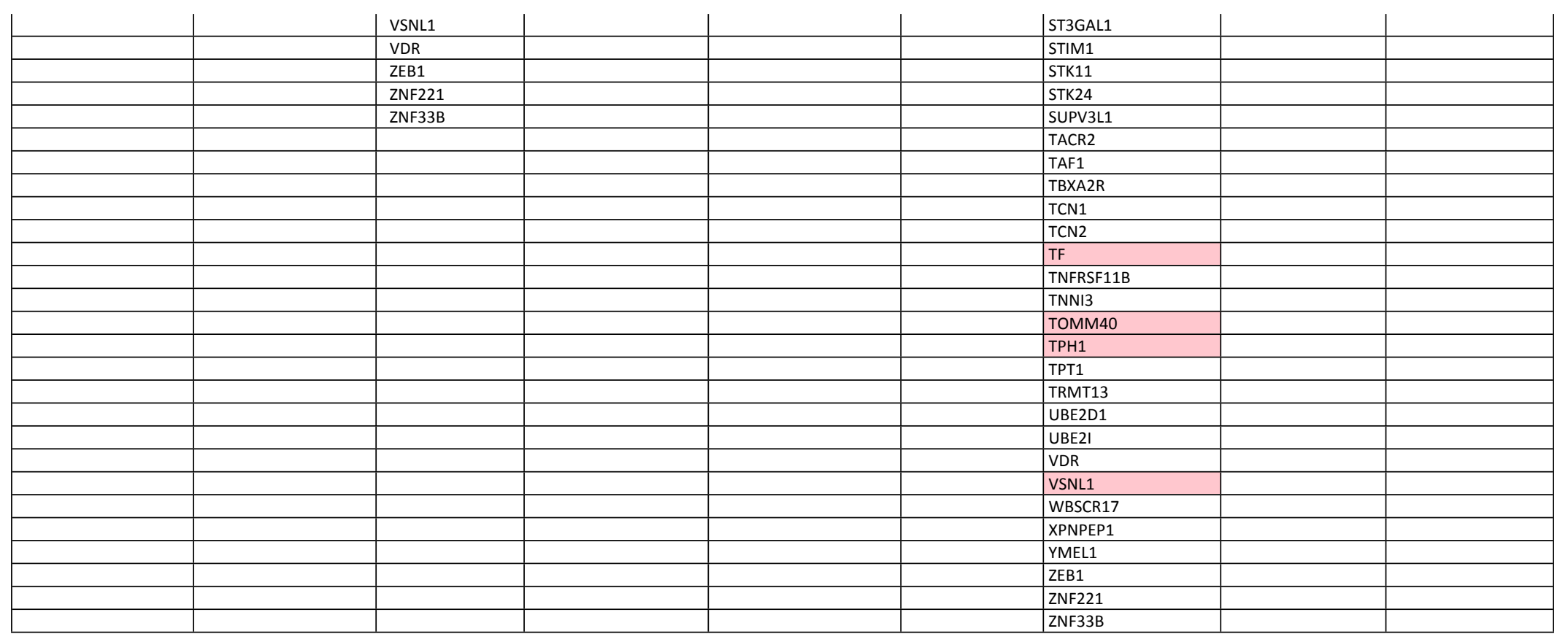

\section{Table S2: List of candidate repurposing anticancer drugs with DrugBank accession no, target name and current indications}

\begin{tabular}{|c|c|c|c|c|c|}
\hline Target & PPI Target & Drug name & DrugBank ID & Current indication & Mode of action \\
\hline APOE & A2M & Cisplatin & DB00515 & metastatic testicular tumors, metastatic ovarian tumors and advanced bladder cancer. & Unknown \\
\hline APP & A2M & Cisplatin & DB00516 & metastatic testicular tumors, metastatic ovarian tumors and advanced bladder cancer. & Unknown \\
\hline \multirow[t]{10}{*}{ APP } & EGFR & Vandetanib & DB05294 & metastatic medullary thyroid cancer & Unknown \\
\hline & & Afatinib & DB08916 & metastatic non-small cell lung cancer & Inhibitor \\
\hline & & Osimertinib & DB09330 & metastatic epidermal growth factor receptor (EGFR) T790M mutation-positive non-small cell lung cancer & Inhibitor \\
\hline & & Gefitinib & DB00317 & metastatic non-small cell lung cancer & Antagonist \\
\hline & & Erlotinib & DB00530 & metastatic non-small cell lung cancer and metastatic pancreatic cancer & Antagonist \\
\hline & & Lapatinib & DB01259 & advanced or metastatic breast cancer & Antagonist \\
\hline & & Neratinib & DB11828 & HER2-overexpressed/amplified breast cancer & Inhibitor \\
\hline & & Brigatinib & DB12267 & anaplastic lymphoma kinase positive, metastatic non-small cell lung cancer & Inhibitor \\
\hline & & Dacomitinib & DB11963 & metastatic non-small cell lung cancer & Inhibitor \\
\hline & & Zanubrutinib & DB15035 & mantle cell lymphoma & Inhibitor \\
\hline APP & JUN & Vincristine & DB00570 & $\begin{array}{l}\text { breast cancer, testicular cancer, lymphomas, neuroblastoma, Hodgkin's and non-Hodgkin's lymphomas, } \\
\text { mycosis fungoides, histiocytosis, and Kaposi's sarcoma. }\end{array}$ & Unknown \\
\hline \multirow[t]{2}{*}{ APP } & KDR & Pazopanib & DB06589 & advanced renal cell cancer and advanced soft tissue sarcoma & Inhibitor \\
\hline & & Regorafenib & DB08896 & metastatic colorectal cancer and metastatic gastrointestinal stromal tumor & Inhibitor \\
\hline
\end{tabular}




\begin{tabular}{|c|c|c|c|c|c|}
\hline & & Nintedanib & DB09079 & metastatic, locally advanced, or locally recurrent non-small cell lung cancer of adenocarcinoma histology & Inhibitor \\
\hline & & Sorafenib & DB00398 & unresectable hepatocellular carcinoma and advanced renal cell carcinoma. & Inhibitor \\
\hline & & Sunitinib & DB01268 & advanced renal cell carcinoma and gastrointestinal stromal tumor & Inhibitor \\
\hline & & Midostaurin & DB06595 & acute myeloid leukemia and mast cell leukemia & Inhibitor, antagonist \\
\hline & & Axitinib & DB06626 & kidney cell cancer & Inhibitor \\
\hline & & Ponatinib & DB08901 & chronic myeloid leukemia and Philadelphia chromosome positive acute lymphoblastic leukemia & Inhibitor \\
\hline & & Lenvatinib & DB09078 & $\begin{array}{l}\text { unresectable hepatocellular carcinoma,advanced renal cell carcinoma and locally recurrent or metastatic, progressive, } \\
\text { radioactive iodine-refractory differentiated thyroid cancer }\end{array}$ & Inhibitor \\
\hline & & Erdafitinib & DB12147 & locally advanced or metastatic urothelial carcinoma & Substrate \\
\hline \multirow[t]{2}{*}{ APP } & MAPT & Paclitaxel & DB01229 & Kaposi's sarcoma and cancer of the lung, ovarian, and breast & Unknown \\
\hline & & Docetaxel & DB01248 & $\begin{array}{l}\text { locally advanced or metastatic breast cancer, locally advanced or metastatic non-small cell lung cancer, } \\
\text { metastatic prostate cancer and gastric adenocarinoma and head and neck cancer }\end{array}$ & Unknown \\
\hline APP & EBP & Tamoxifene & DB00675 & estrogen receptor positive metastatic breast cancer & Inhibitor \\
\hline \multirow[t]{2}{*}{ BIN1 } & MAPT & Paclitaxel & DB01229 & Kaposi's sarcoma and cancer of the lung, ovarian, and breast & Unknown \\
\hline & & Docetaxel & DB01248 & $\begin{array}{l}\text { locally advanced or metastatic breast cancer,locally advanced or metastatic non-small cell lung cancer, } \\
\text { metastatic prostate cancer and gastric adenocarinoma and head and neck cancer. }\end{array}$ & Unknown \\
\hline \multirow[t]{3}{*}{ FYN } & MAPT & Paclitaxel & DB01229 & Kaposi's sarcoma and cancer of the lung, ovarian, and breast & Unknown \\
\hline & & Docetaxel & DB01248 & locally advanced or metastatic breast cancer,locally advanced or metastatic non-small cell lung cancer, & Unknown \\
\hline & & & & metastatic prostate cancer and gastric adenocarinoma and head and neck cancer. & \\
\hline GSK3B & CCND1 & Encorafenib & DB11718 & metastatic melanoma & Inhibitor \\
\hline GSK3B & JUN & Vincristine & DB00570 & $\begin{array}{l}\text { breast cancer, testicular cancer, lymphomas, neuroblastoma, Hodgkin's and non-Hodgkin's lymphomas, } \\
\text { mycosis fungoides, histiocytosis, and Kaposi's sarcoma. }\end{array}$ & Unknown \\
\hline \multirow[t]{7}{*}{ HSPG2 } & FLT1 & Pazopanib & DB06589 & advanced renal cell cancer and advanced soft tissue sarcoma & inhibitor \\
\hline & & Regorafenib & DB08896 & metastatic colorectal cancer, Metastatic Gastrointestinal Stromal Tumor & inhibitor \\
\hline & & Nintedanib & DB09079 & metastatic, locally advanced, or locally recurrent non-small cell lung cancer of adenocarcinoma histology & inhibitor \\
\hline & & Sunitinib & DB01268 & advanced renal cell carcinoma and gastrointestinal stromal tumor & inhibitor \\
\hline & & Axitinib & DB06626 & kidney cell cancer & inhibitor \\
\hline & & Sorafenib & DB00398 & unresectable hepatocellular carcinoma and advanced renal cell carcinoma. & inhibitor \\
\hline & & Lenvatinib & DB09078 & $\begin{array}{l}\text { unresectable hepatocellular carcinoma,advanced renal cell carcinoma and locally recurrent or metastatic, progressive, } \\
\text { radioactive iodine-refractory differentiated thyroid cancer }\end{array}$ & Inhibitor \\
\hline \multirow[t]{10}{*}{ HSPG2 } & KDR & Pazopanib & DB06589 & advanced renal cell cancer and advanced soft tissue sarcoma & Inhibitor \\
\hline & & Regorafenib & DB08896 & metastatic colorectal cancer and metastatic gastrointestinal stromal tumor & Inhibitor \\
\hline & & Nintedanib & DB09079 & metastatic, locally advanced, or locally recurrent non-small cell lung cancer of adenocarcinoma histology & Inhibitor \\
\hline & & Sorafenib & DB00398 & unresectable hepatocellular carcinoma and advanced renal cell carcinoma. & Inhibitor \\
\hline & & Sunitinib & DB01268 & advanced renal cell carcinoma and gastrointestinal stromal tumor & Inhibitor \\
\hline & & Midostaurin & DB06595 & acute myeloid leukemia and mast cell leukemia & Inhibitor, antagonist \\
\hline & & Axitinib & DB06626 & kidney cell cancer & Inhibitor \\
\hline & & Ponatinib & DB08901 & chronic myeloid leukemia and Philadelphia chromosome positive acute lymphoblastic leukemia & Inhibitor \\
\hline & & Lenvatinib & DB09078 & $\begin{array}{l}\text { unresectable hepatocellular carcinoma, advanced renal cell carcinoma and locally recurrent or metastatic, } \\
\text { progressive, radioactive iodine-refractory differentiated thyroid cancer }\end{array}$ & Inhibitor \\
\hline & & Erdafitinib & DB12147 & locally advanced or metastatic urothelial carcinoma & Substrate \\
\hline LRP1 & $\mathrm{A} 2 \mathrm{M}$ & Cisplatin & DB00515 & metastatic testicular tumors, metastatic ovarian tumors and advanced bladder cancer. & Unknown \\
\hline \multirow[t]{8}{*}{ LRP1 } & EGFR & Vandetanib & DB05294 & metastatic medullary thyroid cancer & Unknown \\
\hline & & Afatinib & DB08916 & metastatic non-small cell lung cancer & Inhibitor \\
\hline & & Osimertinib & DB09330 & metastatic epidermal growth factor receptor (EGFR) T790M mutation-positive non-small cell lung cancer & Inhibitor \\
\hline & & Gefitinib & DB00317 & metastatic non-small cell lung cancer & Antagonist \\
\hline & & Erlotinib & DB00530 & metastatic non-small cell lung cancer and metastatic pancreatic cancer & Antagonist \\
\hline & & Lapatinib & DB01259 & advanced or metastatic breast cancer & Antagonist \\
\hline & & Neratinib & DB11828 & HER2-overexpressed/amplified breast cancer & Inhibitor \\
\hline & & Brigatinib & DB12267 & anaplastic lymphoma kinase positive, metastatic non-small cell lung cancer & Inhibitor \\
\hline
\end{tabular}




\begin{tabular}{|c|c|c|c|}
\hline & & Dacomitinib & DB11963 \\
\hline & & Zanubrutinib & DB15035 \\
\hline NECTIN2 & NR4A3 & Dasatinib & DB01254 \\
\hline \multirow[t]{10}{*}{ NRG1 } & EGFR & Vandetanib & DB05294 \\
\hline & & Afatinib & DB08916 \\
\hline & & Osimertinib & DB09330 \\
\hline & & Gefitinib & DB00317 \\
\hline & & Erlotinib & DB00530 \\
\hline & & Lapatinib & DB01259 \\
\hline & & Neratinib & DB11828 \\
\hline & & Brigatinib & DB12267 \\
\hline & & Dacomitinib & DB11963 \\
\hline & & Zanubrutinib & DB15035 \\
\hline \multirow[t]{3}{*}{ NRG1 } & ERBB4 & Afatinib & DB08916 \\
\hline & & Brigatinib & DB12267 \\
\hline & & Zanubrutinib & DB15035 \\
\hline NRG1 & LIMK1 & Dabrafenib & DB08912 \\
\hline PLD3 & NFKB1 & Thalidomide & DB01041 \\
\hline \multirow[t]{4}{*}{ PSEN1 } & BCL2 & Eribulin & DB08871 \\
\hline & & Docetaxel & DB01248 \\
\hline & & Paclitaxel & DB01229 \\
\hline & & Venetoclax & DB11581 \\
\hline \multirow[t]{7}{*}{ SNCA } & ABL1 & Imatinib & DB00619 \\
\hline & & Regorafenib & DB08896 \\
\hline & & Bosutinib & DB06616 \\
\hline & & Dasatinib & DB01254 \\
\hline & & Nilotinib & DB04868 \\
\hline & & Ponatinib & DB08901 \\
\hline & & Brigatinib & DB12267 \\
\hline \multirow[t]{10}{*}{ SNCA } & EGFR & Vandetanib & DB05294 \\
\hline & & Afatinib & DB08916 \\
\hline & & Osimertinib & DB09330 \\
\hline & & Gefitinib & DB00317 \\
\hline & & Erlotinib & DB00530 \\
\hline & & Lapatinib & DB01259 \\
\hline & & Neratinib & DB11828 \\
\hline & & Brigatinib & DB12267 \\
\hline & & Dacomitinib & DB11963 \\
\hline & & Zanubrutinib & DB15035 \\
\hline \multirow[t]{2}{*}{$\mathrm{BCHE}$} & BCHE & Cisplatin & DB00515 \\
\hline & & Irinotecan & DB00762 \\
\hline FYN & FYN & Dasatinib & DB01254 \\
\hline
\end{tabular}

metastatic non-small cell lung cancer

mantle cell lymphoma

acute Lymphoblastic Leukaemias, Chronic Myeloid Leukemia

metastatic medullary thyroid cancer

metastatic non-small cell lung cancer

metastatic epidermal growth factor receptor (EGFR) T790M mutation-positive non-small cell lung cancer

metastatic non-small cell lung cancer

metastatic non-small cell lung cancer and metastatic pancreatic cancer

advanced or metastatic breast cancer

\begin{tabular}{|l|l} 
anaplastic lymphoma kinase positive, metastatic non-small cell lung cancer & Inhibitor
\end{tabular}

\begin{tabular}{|l|l|}
\hline metastatic non-small cell lung cancer & Inhibitor \\
\hline anhibitor & Inhblor \\
\hline
\end{tabular}

\begin{tabular}{|l|l|}
\hline anaplastic lymphoma kinase positive, metastatic non-small cell lung cancer & Inhibitor \\
\hline
\end{tabular}

\begin{tabular}{l|l} 
mantle cell lymphoma & Inhibitor
\end{tabular}

\begin{tabular}{ll} 
metastatic melanoma,anaplastic thyroid cancer & Antagonist, inhibitor \\
\hline multiple myeloma & Antagonist
\end{tabular}

metastatic breast cancer

\begin{tabular}{|ll} 
ocally advanced or metastatic breast cancer, locally advanced or metastatic non-small cell lung cancer, metastatic prostate & Unknown
\end{tabular}

ancer and gastric adenocarinoma and head and neck cancer.

Kaposi's sarcostic adenocarinoma and head and neck cancer.

chronic lymphocytic leukemia or small lymphocytic lymphom

Inhibitor

(iseases,

hypereosinophilic syndrome and/or chronic eosinophilic leukemia, and malignant gastrointestinal stromal tumors.

metastatic colorectal cancer, Metastatic Gastrointestinal Stromal Tumor

chronic, accelerated, or blast phase Philadelphia chromosome-positive $(\mathrm{Ph}+)$ chronic myelogenous leukemia

acute Lymphoblastic Leukaemias, Chronic Myeloid Leukemia

various leukemias, including chronic myeloid leukemia (CML).

accelerated phase chronic myologenic leukemia, Acute Lymphoblastic Leukaemias (ALL)

anaplastic lymphoma kinase positive, metastatic non-small cell lung cancer

metastatic medullary thyroid cancer

\begin{tabular}{l|l} 
metastatic epidermal growth factor receptor (EGFR) T790M mutation-positive non-small cell lung cancer & Inhibitor
\end{tabular}

\begin{tabular}{l|l} 
metastatic non-small cell lung cancer & Antagonist \\
\hline
\end{tabular}

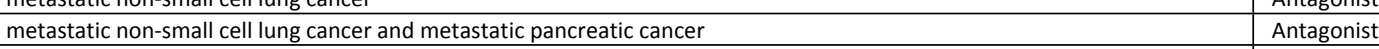

\begin{tabular}{|l|l}
\hline advanced or metastatic breast cancer & Antagonist
\end{tabular}

HER2-overexpressed/amplified breast cancer $\quad$ Inhibitor

anaplastic lymphoma kinase positive, metastatic non-small

metastatic non-small cell lung cancer

metastatic testicular tumors, metastatic ovarian tumors and advanced bladder cancer.

colorectal and advanced pancreatic cancer

Unknown 
Table S3: CoDReS - Computational Drug Repositioning Scores for different drugs

\begin{tabular}{|c|c|c|c|c|c|c|}
\hline A/A & Input Drug & DrugBank ID & Input Score & Functional Score & Structural Score & CoDReS \\
\hline 1 & Erlotinib & DB00530 & 0 & 1 & 0.833333 & 1 \\
\hline 2 & Sunitinib & DB01268 & 0 & 0.100978 & 1 & 0.600534 \\
\hline 3 & Sorafenib & DB00398 & 0 & 0.085432 & 1 & 0.592054 \\
\hline 4 & Imatinib & DB00619 & 0 & 0.060089 & 1 & 0.57823 \\
\hline 5 & Gefitinib & DB00317 & 0 & 0.053233 & 1 & 0.574491 \\
\hline 6 & Vandetanib & DB05294 & 0 & 0.035835 & 1 & 0.565001 \\
\hline 7 & Lenvatinib & DB09078 & 0 & 0.033671 & 1 & 0.563821 \\
\hline 8 & Pazopanib & DB06589 & 0 & 0.021813 & 1 & 0.557353 \\
\hline 9 & Axitinib & DB06626 & 0 & 0.019995 & 1 & 0.556361 \\
\hline 10 & Afatinib & DB08916 & 0 & 0.014282 & 1 & 0.553245 \\
\hline 11 & Dacomitinib & DB11963 & 0 & 0.001818 & 1 & 0.546446 \\
\hline 12 & Nintedanib & DB09079 & 0 & 0.09504 & 0.833333 & 0.506385 \\
\hline 13 & Midostaurin & DB06595 & 0 & 0.017398 & 0.666667 & 0.373126 \\
\hline 14 & Cabozantinib & DB08875 & 0 & 0.004674 & 0.666667 & 0.366186 \\
\hline 15 & Ponatinib & DB08901 & 0 & 0.004155 & 0.666667 & 0.365903 \\
\hline 16 & Neratinib & DB11828 & 0 & 0.002597 & 0.5 & 0.274144 \\
\hline 17 & Lapatinib & DB01259 & 0 & 0.038172 & 0.333333 & 0.202639 \\
\hline
\end{tabular}

Table S4: Physiochemical properties of potential repurposing drugs

\begin{tabular}{|c|c|c|c|c|c|c|c|c|}
\hline Drug name & Mol. Wt. & Rotatable bonds & H-bond acceptor & H-bond donor & TPSA & M log P & BBB penetration \\
\hline Afatinib & 485.94 & 9 & 7 & 2 & 88.61 & 2.43 & No \\
\hline Erlotinib & 393.44 & 10 & 6 & 1 & 74.73 & 1.89 & Yes \\
\hline Gefitinib & 446.9 & 8 & 7 & 1 & 68.74 & 2.82 & Yes \\
\hline Imatinib & 439.6 & 8 & 6 & 2 & 86.28 & 2.15 & No \\
Sunitinib & 398.47 & 8 & 4 & 3 & 77.23 & 2.06 & Yes \\
\hline Vandetanib & 475.35 & 6 & 6 & 1 & 59.51 & 3.45 & Yes \\
\hline
\end{tabular}

$\begin{array}{llcc}\text { Vandetanib } & 475.35 & 6 & 6 \\ \text { *Highlighted drugs have good drug-likeness and BBB permeation }\end{array}$ 
Table S5: List of AD-related miRNAs, Drugs and their targets

\begin{tabular}{|l|l|l|}
\hline AD related miRNA & Targets & Drugs \\
\hline mir-103a & VEGFA & Vandetanib \\
\hline mir-103a & RET & Vandetanib \\
\hline mir-103a-1 & PTK6 & Vandetanib \\
\hline mir-103a-2 & PTK6 & Vandetanib \\
\hline mir-106b & VEGFA & Vandetanib \\
\hline mir-106b & VEGFA & Vandetanib \\
\hline mir-107 & VEGFA & Vandetanib \\
\hline mir-107 & RET & Vandetanib \\
\hline mir-1285 & RET & Vandetanib \\
\hline mir-138 & PTK6 & Vandetanib \\
\hline mir-139 & TEK & Vandetanib \\
\hline mir-144 & TEK & Vandetanib \\
\hline mir-144 & RET & Vandetanib \\
\hline mir-148b & TEK & Vandetanib \\
\hline mir-15a & VEGFA & Vandetanib \\
\hline mir-15a & RET & Vandetanib \\
\hline mir-15b & VEGFA & Vandetanib \\
\hline mir-15b & RET & Vandetanib \\
\hline mir-16 & VEGFA & Vandetanib \\
\hline mir-16 & RET & Vandetanib \\
\hline mir-16-2 & TEK & Vandetanib \\
\hline mir-17 & VEGFA & Vandetanib \\
\hline mir-186 & VEGFA & Vandetanib \\
\hline mir-195 & VEGFA & Vandetanib \\
\hline mir-195 & RET & Vandetanib \\
\hline mir-200a & EGFR & Vandetanib \\
\hline mir-200a & EGFR & Erlotinib \\
\hline mir-200a & EGFR & Gefitinib \\
\hline mir-200a & EGFR & Erlotinib \\
\hline mir-200a & EGFR & Gefitinib \\
\hline mir-200c & VEGFA & Vandetanib \\
\hline mir-200c & RET & Vandetanib \\
\hline mir-206 & VEGFA & Vandetanib \\
\hline mir-20a & VEGFA & Vandetanib \\
\hline mir-27a & EGFR & Vandetanib \\
\hline mir-27a & EGFR & Gefitinib \\
\hline mir-27a & EGFR & Erlotinib \\
\hline mir-27a & EGFR & Gefitinib \\
\hline & & \\
\hline
\end{tabular}




\begin{tabular}{|l|l|l|} 
mir-27a & EGFR & Erlotinib \\
\hline mir-29a & VEGFA & Vandetanib \\
\hline mir-29b & VEGFA & Vandetanib \\
\hline mir-29b & RET & Vandetanib \\
\hline mir-29b-2 & VEGFA & Vandetanib \\
\hline mir-29c & VEGFA & Vandetanib \\
\hline mir-301a & RET & Vandetanib \\
\hline mir-339 & VEGFA & Vandetanib \\
\hline mir-342 & VEGFA & Vandetanib \\
\hline mir-613 & VEGFA & Vandetanib \\
\hline mir-766 & RET & Vandetanib \\
\hline mir-885-3p & EGFR & Vandetanib \\
\hline mir-885-3p & EGFR & Erlotinib \\
\hline mir-885-3p & EGFR & Gefitinib \\
\hline mir-93 & VEGFA & Vandetanib \\
\hline
\end{tabular}

